\title{
THE MACKEY PROBLEM FOR THE COMPACT-OPEN TOPOLOGY
}

BY

\author{
ROBERT F. WHEELER
}

\begin{abstract}
Let $C_{c}(T)$ denote the space of continuous real-valued functions on a completely regular Hausdorff space $T$, endowed with the compactopen topology. Well-known results of Nachbin, Shirota, and Warner characterize those $T$ for which $C_{c}(T)$ is bornological, barrelled, and infrabarrelled. In this paper the question of when $C_{c}(T)$ is a Mackey space is examined. A convex strong Mackey property (CSMP), intermediate between infrabarrelled and Mackey, is introduced, and for several classes of spaces (including first countable and scattered spaces), a necessary and sufficient condition on $T$ for $C_{c}(T)$ to have CSMP is obtained.
\end{abstract}

Let $T$ be a completely regular Hausdorff space, and let $C_{c}(T)$ denote the space of real-valued continuous functions on $T$, endowed with the compact-open topology. The problem of relating topological properties of $T$ to linear topological properties of $C_{c}(T)$ has been investigated by many prominent mathematicians. For example, Nachbin [22] and Shirota [28] used this approach to find the first example of a barrelled locally convex space which is not bornological. Many additional results were furnished by Warner [31]; in particular, he characterized those spaces $T$ for which $C_{c}(T)$ is complete, separable, or infrabarrelled. More recently, Buchwalter (many papers, enumerated in [3]), De Wilde and Schmets [6], Haydon [15], [16], and many others have provided new insights into the relationship between $T$ and $C_{c}(T)$.

One problem left largely untouched by these authors is the following: find necessary and sufficient conditions on $T$ for $C_{c}(T)$ to be a Mackey space. Paraphrasing a remark of Conway [5] in a slightly different setting it is difficult to find locally convex spaces with intrinsically defined topologies which are Mackey spaces, except by virtue of some formally stronger property (such as metrizable, barrelled, or bornological). A well-known example of Conway (suit-

Received by the editors June 20, 1975.

AMS (MOS) subject classifications (1970). Primary 46E10, 54C35; Secondary 46A05, $60 \mathrm{B05}$.

Key words and phrases. Bounded set, compact-open topology, countably compact, convex strong Mackey property, infrabarrelled space, Mackey space, strict topology, Warner bounded set. 
ably reinterpreted) shows that $C_{c}(T)$ is not always a Mackey space. Beyond this, little seems to have appeared in the literature.

In this paper we investigate the Mackey problem for $C_{c}(T)$ in some detail. We are led to consider a convex strong Mackey property (CSMP) for locally convex spaces, intermediate between infrabarrelled and Mackey, and for certain $T$ (in particular, for first countable spaces and scattered spaces) we obtain a necessary and sufficient condition on $T$ for $C_{c}(T)$ to possess CSMP. Throughout the emphasis is on subsets of the dual of $C_{c}(T)$ with various compactness properties, and the topological properties which their supports (subsets of $T$ ) possess.

1. Basic definitions and results. The reader may consult Kelley and Namioka [18] or Schaefer [26] for important concepts in the theory of locally convex Hausdorff topological vector spaces (LCS), in particular the definitions of barrelled, infrabarrelled, and Mackey spaces. If $E$ is a LCS, then $E^{\prime}$ is the space of continuous linear functionals on $E$, and $\sigma\left(E^{\prime}, E\right)$ and $\beta\left(E^{\prime}, E\right)$ denote the weak* and strong topologies on $E^{\prime}$, respectively.

Two "strong Mackey" properties have appeared recently in the literature of the strict topology $\beta$ of Buck [4]; see, in particular, Conway [5]. These are obtained by replacing "weak*-compact convex and circled" in the usual statement of the Mackey property by "weak*-compact" and "relatively weak*-countably compact" respectively. We modify these as follows.

Definition 1.1. A LCS $E$ has the convex strong Mackey property (CSMP) if every weak*-countably compact convex circled subset of $E^{\prime}$ is equicontinuous.

Here $H \subset E^{\prime}$ is weak*-countably compact if every sequence in $H$ has a weak*-cluster point in $H$.

THEOREM 1.2. Every infrabarrelled LCS has CSMP. Every LCS with CSMP is a Mackey space.

ProOF. Corollary 10.3 of [18] implies that every weak*-countably compact convex circled subset of $E^{\prime}$ is strongly bounded. This proves the first assertion; the second is trivial.

Both converses fail; we give $C_{c}(T)$ examples below (see 3.6 and 3.7).

The literature of the compact-open topology through 1972 is admirably summarized in the papers of Warner [31] and Buchwalter[3]. The GillmanJerison text [9] is another valuable source of information about $C(T)$. We shall use some of the terminology of the French school to describe subsets of $T$. A subset $B$ of $T$ is bounded in $T$ if whenever $f \in C(T)$, then the restriction of $f$ to $B$ is bounded. Any pseudocompact subset of $T$ is bounded in $T$; the converse fails, but a closed bounded subset of a normal space is pseudocompact. 
A subset $B$ of $T$ is Warner bounded in $T$ if whenever $A \subset C(T)$ is bounded in the compact-open topology, then $\sup \{|f(t)|: f \in A, t \in B\}<\infty$ (see [3, Proposition 1.4.1] for other characterizations). We have relatively compact $\rightarrow$ Warner bounded $\rightarrow$ bounded.

A space $T$ is said to be a Warner space if every bounded set is Warner bounded; this is true, for example, if $T$ is a $k_{R}$-space (defined by (3) of Theorem 1 in [31]), and therefore holds for locally compact and first countable spaces. Also $T$ is a $\mu$-space if every bounded subset of $T$ is relatively compact; this holds if $T$ is realcompact (= a $Q$-space), but not conversely $[9,9 \mathrm{~L}]$.

With these definitions we can (following Buchwalter) restate two of the classic results of the theory of $C_{c}(T)$ as follows:

Proposition 1.3 (Nachbin, Shirota). $C_{c}(T)$ is barrelled if and only if every bounded subset of $T$ is relatively compact.

PROPOSITION 1.4 (WARNER). $C_{c}(T)$ is infrabarrelled if and only if every Warner bounded subset of $T$ is relatively compact.

The characterizations of the convex strong Mackey property for $C_{c}(T)$ obtained below are of the same general type as these.

The dual space of $C_{c}(T)$ can be represented as $M_{c}(T)$, the space of regular Borel measures on $T$ with compact support. If $\mu \in M_{c}(T)$, the support of $\mu$ is $\operatorname{spt}(\mu)=\{t \in T$ : for each open set $U$ containing $t,|\mu|(U)>0\}$. If $H \subset M_{c}(T)$, the support of $H$ is the closure of $\bigcup_{\mu \in H} \operatorname{spt}(\mu)$. If $t \in T, \delta(t)$ is the point mass at $t$.

Let $C^{*}(T)$ denote the subspace of $C(T)$ consisting of all bounded realvalued continuous functions on $T$. Then $C^{*}(T)$ is dense in $C_{c}(T)$, and so $M_{c}(T)$ is also the dual of $C^{*}(T)$ with the relative compact-open topology. The phrase "weak*.", applied to $M_{c}(T)$, will always refer to the $\sigma\left(M_{c}(T), C(T)\right)$-topology; the $\sigma\left(M_{c}(T), C^{*}(T)\right)$-topology on $M_{c}(T)$, when used, will always be indicated in this specific way (or as $\sigma\left(M_{c}, C^{*}\right)$ if $T$ is understood).

Now $C^{*}(T)$ with the supremum norm is a Banach space, and $M_{c}(T)$ embeds naturally in its Banach space dual $(\|\mu\|=|\mu|(T)$ coincides with the usual dual norm). Hence for a subset $H$ of $M_{c}(T), \sigma\left(M_{c}, C\right)$-bounded $\rightarrow \sigma\left(M_{c}, C^{*}\right)$-bounded $\longleftrightarrow$ uniformly bounded. If $\operatorname{spt}(H)$ is compact (or even bounded in $T$ ), all three conditions are equivalent.

Proposition 1.5 ([31, Lemma 4], [8, p. 117]). A subset $H$ of $M_{c}(T)$ is equicontinuous with respect to the compact-open topology if and only if (1) $\operatorname{spt}(H)$ is compact; and (2) sup $\{\|\mu\|: \mu \in H\}<\infty$. Here (2) may be replaced by: $\left(2^{\prime}\right) H$ is $\sigma\left(M_{c}, C\right)$-bounded. 
The finest locally convex topology on $C^{*}(T)$ which agrees with the compact-open topology on norm-bounded sets has been examined by many authors. Sentilles [27] refers to it as the substrict topology $\beta_{0}$ and gives a number of characterizations. If $T$ is locally compact, $\beta_{0}$ coincides with the strict topology $\beta$ of Buck [4]. In general the dual space of $\left(C^{*}(T), \beta_{0}\right)$ is $M_{t}(T)$, the space of bounded compact-regular Borel measures on $T$. The space $M_{c}(T)$ embeds in $M_{t}(T)$, and indeed the latter is the Banach space completion of the former. The importance of $\beta_{0}$ for our purposes lies in the following result of Gulick [13].

Proposition 1.6 (GuLICK). If every $\sigma$-compact subset of $T$ is relatively compact, then (1) $C^{*}(T)=C(T)$ and (2) the substrict and compact-open topologies on $C^{*}(T)$ coincide.

The topological property in Proposition 1.6 has been used by Warner [31, Theorem 12] and Gulick [11], [12] , [13]; some writers refer to it as shamcompactness. For example, $\left[0, \omega_{1}\right)$, the space of ordinals less than the first uncountable ordinal, is sham-compact but not compact. We will have several occasions to use Proposition 1.6 in connection with existing results on the strict topology.

2. Supports of sets of measures. In this section we prove that the support of a weak*-countably compact convex circled subset of $M_{c}(T)$ must posses certain topological properties. These properties are related to such familiar concepts as pseudocompactness and countable compactness, and also to the notions of boundedness and Wamer boundedness. We give the basic definitions, one of which is already in the literature, and then summarize the relationships among these concepts.

Definition 2.1 [10]. A space $D$ is $\aleph_{0}$-bounded if every countable (equivalently, separable) subset is relatively compact in $D$.

Definition 2.2. A space $D$ is almost $\aleph_{0}$-bounded if for every sequence $\left(G_{n}\right)$ of nonempty $G_{\delta}$-subsets of $D$ there is a sequence $\left(y_{n}\right)$, with $y_{n} \in G_{n} \forall n$, such that the set consisting of all $y_{n}$ is relatively compact in $D$.

Since every nonempty $G_{\delta}$ in a completely regular $T_{2}$ space contains a nonempty zero-set, " $G_{\delta}$-subsets" may be replaced by "zero-sets" in 2.2.

REMARK 2.3. If $D$ is a subset of $T$, then the following diagram summarizes relationships among the various topological properties of $D$ which have been described. Note that "bounded" and "Warner bounded" are relative properties of $D$ (depending on its position in $T$ ), while the others are absolute properties of $D$.

All of these implications are well-known or easily established. For example, (3) $\rightarrow$ (5): if $D$ is not Warner bounded, let $A$ be a subset of $C(T)$, bounded in the compact-open topology, such that $\sup \{|f(t)|: f \in A, t \in D\}=\infty$. Choose 
(1)

(3)

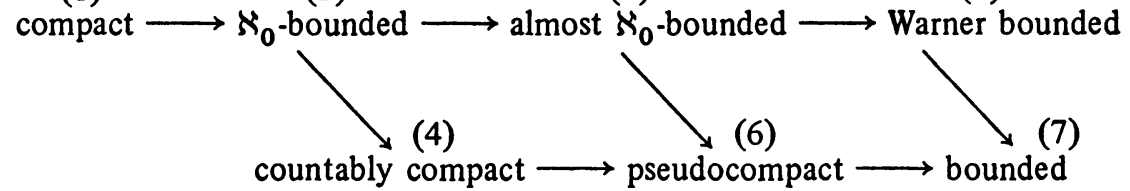

sequences $\left(f_{n}\right)$ in $A$ and $\left(t_{n}\right)$ in $D$ such that $\left|f_{n}\left(t_{n}\right)\right|>n$ for all $n$. Let $G_{n}=$ $\left\{t \in D:\left|f_{n}(t)\right|=\left|f_{n}\left(t_{n}\right)\right|\right\}$. Then each $G_{n}$ is a nonempty $G_{\delta}$-subset of $D$, but it is not possible to choose $y_{n} \in G_{n}$ such that the set of all $y_{n}$ is relatively compact (in $D$ or in $T$ ), since this would contradict the boundedness of $A$.

No other implication holds among these properties, as the following list of examples shows:

2.3.1. $T=D=\left[0, \omega_{1}\right)$. Then $D$ satisfies $2,3,4,5,6$, and 7 , but not 1 .

2.3.2. $T=D=\beta N-\{p\}$, where $p$ is any point in $\beta N-N$. Then $D$ satisfies $4,5,6$, and 7 , but not 1,2 , and 3 (for 3 , let $G_{n}=\{n\}$ ).

2.3.3. $T=D=$ the Tychonoff plank $[9,8.20]$. Then $D$ satisfies $3,5,6$, and 7 , but not 1,2 , and 4 .

2.3.4. $T=D=$ an infinite countably compact space in which compact sets are finite (see [23] for an example). Then $D$ satisfies 4,6 , and 7 , but not $1,2,3$, and 5 .

2.3.5. $T=$ the Tychonoff plank, $D=\left\{\left(\omega_{1}, n\right): n \in N\right\}$ is the right edge of $T$. Then $D$ satisfies 5 and 7 , but not $1,2,3,4$, and 6 .

Under special assumptions on $D$ and $T$, many of these implications can be reversed. For example, if $T$ is normal and $D$ is closed in $T$, then 4,6 , and 7 are equivalent. If every point of $T$ is a $G_{\delta}$ (in particular, if $T$ is first countable), then 2 and 3 are equivalent. And if $T$ is metrizable, $1,2,3,4$, and 6 are equivalent, while 5 and 7 are equivalent to relative compactness of $D$.

We now proceed to measure theoretic results. It follows from the results of Nachbin, Shirota, and Warner that

(1) if $H \subset M_{c}(T)$ is $\sigma\left(M_{c}, C\right)$-bounded, then spt $(H)$ is a bounded subset of $T$;

(2) if $H \subset M_{c}(T)$ is $\beta\left(M_{c}, C\right)$-bounded, then $\operatorname{spt}(H)$ is a Warner bounded subset of $T$.

Both will hold if $H$ is weak*-countably compact convex and circled (as in 1.2).

In the other direction, we have an example due to Conway [5, p. 481]; Proposition 1.6 applies.

Example 2.4 (Conway). Let $T=\left[0, \omega_{1}\right)$, and let $H$ be the weak*. closed convex circled hull of $\{1 / 2(\delta(s)-\delta(s+1)): s$ a countable nonlimit ordinal $\}$ in $M_{c}(T)$. Then $H$ is weak*-compact convex and circled, and $\operatorname{spt}(H)=T$ is $\aleph_{0}$ bounded, but not compact. Hence $C_{c}(T)$ is not a Mackey space. 
A similar example is noted by Morris and Wulbert [20, p. 327].

Our basic results are as follows:

THEOREM 2.5. If $D$ is an $\aleph_{0}$-bounded subset of $T$, then there is a weak*countably compact convex circled subset $H$ of $M_{c}(T)$ such that $D \subset \operatorname{spt}(H)$.

THEOREM 2.6. If $H \subset M_{c}(T)$ is weak*-countably compact and convex, then $\operatorname{spt}(H)$ is almost $\aleph_{0}$-bounded.

ProOF OF 2.5. Let $H$ be the set of measures $\mu \in M_{c}(T)$ such that (1) $\|\mu\| \leqslant 1$; and (2) there is a countable subset of $D$ whose closure (a compact subset of $D)$ contains $\operatorname{spt}(\mu)$. Then $H$ is convex and circled. Let $\left(\mu_{n}\right)$ be a sequence in $H$. Then there is a countable subset of $T$ of $D$ whose closure $F$ contains $\bigcup_{n=1}^{\infty} \operatorname{spt}\left(\mu_{n}\right)$. Each $\mu_{n}$, when restricted to the Borel sets of $F$, determines a member $\lambda_{n}$ of the closed unit ball of $M(F)$, the Banach space dual of $C(F)$. By Alaoglu's Theorem, $\left(\lambda_{n}\right)$ has a $\sigma(M(F), C(F))$-cluster point $\lambda_{0}$ in $M(F)$, with $\left\|\lambda_{0}\right\| \leqslant 1$. Defining $\mu_{0}(E)=\lambda_{0}(E \cap F)$ for each Borel set $E$ in $T$, we have $\mu_{0}$ $\in M_{c}(T)$ (indeed $\mu_{0} \in H$, since $\operatorname{spt}\left(\mu_{0}\right) \subset F$ ), and $\mu_{0}$ is a $\sigma\left(M_{c}(T), C(T)\right.$ )cluster point of $\left(\mu_{n}\right)$. Hence $H$ is weak*-countably compact.

PROOF OF 2.6. Let $\left(G_{n}\right)$ be a sequence of nonempty $G_{\delta}$-subsets of $\operatorname{spt}(H)$ (with the relative topology of $T$ ). Let $F_{n}$ be a $G_{\delta}$-subset of $T$ such that $\operatorname{spt}(H)$ $\cap F_{n}=G_{n}$; write $F_{n}=\bigcap_{k=1}^{\infty} W_{n, k}$, where each $W_{n, k}$ is open in $T$. Let $x_{n}$ be a fixed member of $G_{n}$. For each $n$ and $k$, choose a cozero-set $U_{n, k}$ and a closed set $Z_{n, k}$ of $T$ with $x_{n} \in U_{n, k} \subset Z_{n, k} \subset W_{n, k}$. We may assume that $Z_{n, k} \supset$ $Z_{n, k+1}$ for all $n$ and $k$.

Let $H_{n, k}=\left\{\mu \in H:|\mu|\left(U_{n, k}\right)=0\right\}$. It follows from [30, Theorem 3, p. 183] that each $H_{n, k}$ is $\sigma\left(M_{c}, C^{*}\right)$-closed in $H$, and therefore $\sigma\left(M_{c}, C\right)$-closed in $H$. Suppose $\bigcup_{n, k} H_{n, k}=H$. Since every countably compact space satisfies the Baire Category Theorem (see 2.9 below), some $H_{n_{0}, k_{0}}$ will contain a relative weak*-interior point $\mu_{0}$.

Now $\bigcup_{\mu \in H} \operatorname{spt}(\mu)$ is dense in $\operatorname{spt}(H)$, so there is a member $\mu_{1}$ of $H$ and a point $z_{1} \in U_{n_{0}, k_{0}} \cap \operatorname{spt}\left(\mu_{1}\right)$. Then $\lambda \mu_{0}+(1-\lambda) \mu_{1} \in H$ for $0 \leqslant \lambda \leqslant 1$, and so there is a $\lambda_{0}<1$ such that $\lambda \mu_{0}+(1-\lambda) \mu_{1} \in H_{n_{0}, k_{0}}$ for $\lambda_{0}<\lambda<1$ (since $\mu_{0}$ is a relative weak*-interior point of $\left.H_{n_{0}, k_{0}}\right)$. But this is absurd, since

$$
\left|\lambda \mu_{0}+(1-\lambda) \mu_{1}\right|\left(U_{n_{0}, k_{0}}\right)=(1-\lambda)\left|\mu_{1}\right|\left(U_{n_{0}, k_{0}}\right)>0 \text { for } \lambda<1 \text {. }
$$

Thus there is a measure $\bar{\mu}$ in $H \backslash \bigcup_{n, k} H_{n, k}$. This implies that $|\bar{\mu}|\left(Z_{n, k}\right) \geqslant$ $|\bar{\mu}|\left(U_{n, k}\right)>0 \forall n, k$. For fixed $n,\left\{Z_{n, k} \cap \operatorname{spt}(\bar{\mu})\right\}_{k=1}^{\infty}$ is a decreasing nonempty family of compact sets, and so there is a point $y_{n} \in \operatorname{spt}(\bar{\mu}) \cap \bigcap_{k=1}^{\infty} Z_{n, k}$ $\subset \operatorname{spt}(H) \cap F_{n}=G_{n}$. The set consisting of all $y_{n}$ is relatively compact in $\operatorname{spt}(H)$ because it is a subset of $\operatorname{spt}(\bar{\mu})$. 
COROLlARY 2.7. (a) If $H \subset M_{c}(T)$ is weak*-countably compact and convex, then $\operatorname{spt}(H)$ is Warner bounded and pseudocompact. (b) If $T$ is normal or countably paracompact, then $\operatorname{spt}(H)$ is countably compact. (c) If every point of $T$ is $a G_{\delta}$, then $\operatorname{spt}(H)$ is $\aleph_{0}$-bounded.

Proof. (a) and (c) follow from Remark 2.3 and Theorem 2.6. Normality and countable paracompactness are preserved by closed subspaces, and each, in the presence of pseudocompactness, implies countable compactness, so (b) holds. (In the statement of (b), normality can be weakened to the property in 3L.4 of [9], while countable paracompactness can be weakened to the ss-discrete property of Isawata [17, p. 147].)

COROLLARY 2.8. If $T$ is metacompact and normal, countably paracompact, or first countable, then $C_{c}(T)$ has CSMP.

Proof. In view of Corollary 2.7 , this is an immediate consequence of the Arens-Dugundji Theorem [7, p. 229] and Proposition 1.5. In the normal case, a stronger result is available: since a metacompact normal space is a $\mu$ space, $C_{c}(T)$ is barrelled. Also metacompactness may be replaced by the property of isocompactness studied by Bacon [2] .

REMARK 2.9. The Baire Category Theorem is valid for any pseudocompact space (use the standard argument, combined with Lemma 9.13 of [9]). Thus Theorem 2.6 yields the following symmetric corollary: If $H$ is a weak*-pseudocompact convex subset of $M_{c}(T)$, then $\operatorname{spt}(H)$ is pseudocompact.

3. Some special classes of spaces. The results of $\$ 2$ suggest the following conjecture:

Conjecture 3.1. $C_{c}(T)$ has CSMP if and only if every $\aleph_{0}$-bounded subset of $T$ is relatively compact.

This has the virtue of resembling the classical results of Propositions 1.3 and 1.4. We have no proof of this conjecture in the general case (nor any counterexample), but we do verify it for two substantial classes of spaces: spaces in which every point is a $G_{\delta}$, and almost discrete spaces (defined below). Under special set theoretic assumptions, we also show that $C_{c}(T)$ has CSMP when every closed subset of $T$ is a $G_{\delta}$.

THEOREM 3.2. If every point of $T$ is $a G_{\delta}$, then Conjecture 3.1 is valid.

Proof. The necessity in Conjecture 3.1 is valid for any completely regular Hausdorff $T$. Indeed if $D$ is an $\aleph_{0}$-bounded subset of $T$, then, by Theorem 2.5, $D \subset \operatorname{spt}(H)$, where $H$ is equicontinuous. Hence $D$ is relatively compact, by Proposition 1.5. 
Now assume that every point of $T$ is a $G_{\delta}$. If $H \subset M_{c}(T)$ is weak*-countably compact convex and circled, then $H$ is weak*-bounded, and $\operatorname{spt}(H)$ is closed and $\aleph_{0}$-bounded, by Corollary 2.7 (c). Thus the sufficiency in Conjecture 3.1 follows from Proposition 1.5.

Let us say that $T$ is almost discrete if every compact subset of $T$ has an isolated point. This term has been used by Babiker [1]; the following proposition (easily established using results of W. Rudin [25] and Knowles [19]) relates our property to his.

Proposition 3.3. A space $T$ is almost discrete if and only if $M_{t}(T)=l^{1}(T)$; i.e., every member of $M_{t}(T)$ is of the form $\Sigma_{n=1}^{\infty} c_{n} \delta\left(t_{n}\right)$, where $\Sigma_{n=1}^{\infty}\left|c_{n}\right|<\infty$ and $\left(t_{n}\right)$ is a sequence in $T$.

Almost discreteness is weaker than the familiar property of being scattered (i.e., every subset has an isolated point). For example, if $T$ is the real line with the right half-open interval topology, then $T$ is almost discrete since every compact subset of $T$ is countable, but $T$ has no isolated points. The space $\left[0, \omega_{1}\right]$ is an uncountable compact space which is almost discrete.

LEMMA 3.4. Let $T$ be almost discrete. If $H \subset M_{c}(T)$ is convex, circled, weak ${ }^{*}$-bounded, and norm-complete, then $D=\bigcup_{\mu \in H}$ spt $\mu$ is $\aleph_{0}$-bounded.

Proof. Let $\left(y_{n}\right)$ be a sequence in $D$, and choose a sequence $\left(\mu_{n}\right)$ in $H$ such that $y_{n} \in \operatorname{spt}\left(\mu_{n}\right) \forall n$. This implies (by Proposition 3.3) that $\mu_{n}\left(\left\{y_{n}\right\}\right) \neq$ $0 \forall n$. Choose a sequence $\left(\alpha_{n}\right)$ of nonnegative numbers by induction as follows: for $n=1$, let $\alpha_{1}=1 / 2$. Assume $\alpha_{1}, \alpha_{2}, \ldots, \alpha_{n}$ have been chosen so that (1) $0 \leqslant \alpha_{i} \leqslant 1 / 2^{i}$ for $1 \leqslant i \leqslant n$, and $(2)\left|\left(\sum_{i=1}^{m} \alpha_{i} \mu_{i}\right)\left(\left\{y_{j}\right\}\right)\right|>1 / 2\left|\left(\sum_{i=1}^{j} \alpha_{i} \mu_{i}\right)\left(\left\{y_{j}\right\}\right)\right|$ $>0$ for $1 \leqslant j \leqslant m \leqslant n$. If $\left(\sum_{i=1}^{n} \alpha_{i} \mu_{i}\right)\left(\left\{y_{n+1}\right\}\right) \neq 0$, let $\alpha_{n+1}=0$. Otherwise, choose $\alpha_{n+1}$ positive, but so small that (1) $0<\alpha_{n+1}<1 / 2^{n+1}$, and (2) $\left|\left(\sum_{i=1}^{n+1} \alpha_{i} \mu_{i}\right)\left(\left\{y_{j}\right\}\right)\right|>1 / 2\left|\left(\Sigma_{i=1}^{j} \alpha_{i} \mu_{i}\right)\left(\left\{y_{j}\right\}\right)\right|$ for $1 \leqslant j \leqslant n$. This describes the procedure, by induction.

Now $\sum_{n=1}^{\infty} \alpha_{n} \leqslant 1$, and $H$ is uniformly bounded in $M_{c}(T)$, by the remarks preceding Proposition 1.5. Thus $\Sigma_{n=1}^{\infty} \alpha_{n} \mu_{n}$ is norm-convergent to a measure $\mu_{0}$ in $M_{t}(T)$ (since $M_{t}$ is a Banach space). Since $H$ is convex, circled, and normcomplete, $\mu_{0} \in H \subset M_{c}(T)$. Also for any $j$,

$$
\left|\mu_{0}\left(\left\{y_{j}\right\}\right)\right|=\lim _{n \rightarrow \infty}\left|\left(\sum_{i=1}^{n} \alpha_{i} \mu_{i}\right)\left(\left\{y_{j}\right\}\right)\right| \geqslant \frac{1}{2}\left|\left(\sum_{i=1}^{j} \alpha_{i} \mu_{i}\right)\left(\left\{y_{j}\right\}\right)\right|>0 .
$$

Thus $y_{j} \in \operatorname{spt}\left(\mu_{0}\right) \forall j$, and so the set of all $y_{j}$ is relatively compact in $D$.

THEOREM 3.5. If $T$ is almost discrete, then Conjecture 3.1 is valid.

Proof. Since $\operatorname{spt}(H)$ is the closure of $\bigcup_{\mu \in H} \operatorname{spt}(\mu)$, and a weak*. 
countably compact convex circled set satisfies the hypothesis of Lemma 3.4, a proof similar to that of Theorem 3.2 can be given.

EXAMPLE 3.6. Let $T$ denote the Tychonoff plank $[9,8.20]$, and let $S=$ $T \backslash\left\{\left(\alpha, \omega_{0}\right): \alpha\right.$ is a countable limit ordinal $\}$. The space $S$ was considered by Shirota [28]. Both $S$ and $T$ are almost discrete. The right-hand edge of each is a Warner bounded subset which is not relatively compact; hence, by Proposition 1.4, neither $C_{c}(S)$ nor $C_{c}(T)$ is infrabarrelled. The subset $\left[0, \omega_{1}\right) \times\left[0, \omega_{0}\right)$ of $T$ is $\aleph_{0}$-bounded but not relatively compact in $T$; hence, by Theorem $3.5, C_{c}(T)$ does not have CSMP (Conway's example (2.4) can be modified to show that $C_{c}(T)$ is not even a Mackey space). On the other hand, it can be checked that every $\aleph_{0}$-bounded subset of $S$ is relatively compact in $S$; hence $C_{c}(S)$ has CSMP.

EXAMPLE 3.7. Let $P$ be the set of countable ordinals with the discrete topology, and let $X$ be the union of all closures of countable subsets of $P$ in $\beta P$. Then $X$ is sham-compact locally compact; hence (appealing to Proposition 1.6) Example 3.4 of [33] shows that $C_{c}(X)$ is Mackey. However, $X$ is $\aleph_{0}$-bounded, and the proof of Theorem 2.5 (with $D=X=T$ ) shows that the closed unit ball $H$ of $M_{c}(X)$ is weak*-countably compact convex and circled. Since $\operatorname{spt}(H)=X$ is not compact, $C_{c}(X)$ does not have CSMP.

We conclude this section with an application of recent consistency results in set-theoretic topology. See [24] or [29] for a discussion of Martins's Axiom and its significance.

\section{THEOREM 3.8. Assume Martin's Axiom and the negation of the Con-} tinuum Hypothesis. (a) If every closed subset of $T$ is a $G_{\delta}$, then $C_{c}(T)$ has CSMP. (b) If $T$ is perfectly normal, then $C_{c}(T)$ is infrabarrelled.

Proof. (a) If $H \subset M_{c}(T)$ is weak*-countably compact convex and circled, then $\operatorname{spt}(H)$ is a countably compact space (by 2.7 (c)) in which closed sets are $G_{\delta}$ 's. But under the stated set-theoretic assumptions, Weiss [32] has shown that such a space is always compact. Thus the result follows from Proposition 1.5. (b) The proof is similar to that of (a); use remark (2) preceding Example 2.4, and the fact that a closed Warner bounded subset of a normal space is countably compact.

EXAMPle 3.9. Assuming a different set-theoretic axiom $(V=L)$, which implies the Continuum Hypothesis, Ostaszewski has obtained a result opposite to that of Weiss by constructing a perfectly normal, first countable, hereditarily separable, countably compact, noncompact space $O$ (see Chapter 6 of [24] for details). Since $O$ is a Warner bounded subset of itself, $C_{c}(O)$ is not infrabarrelled, by Proposition 1.4. Thus the assertion of $3.8(\mathrm{~b})$ is independent of the Zermelo-Fraenkel axioms of set theory; i.e., it can neither be proved nor disproved without additional assumptions. 
On the other hand, the conclusion of Theorem 3.8 (a) remains valid for $C_{c}(O) ; O$ is first countable, and hereditary separability implies that every $\aleph_{0}$ bounded subset is compact. Thus Theorem 3.2 applies, and $C_{c}(O)$ has CSMP. This leaves open the possibility that Theorem 3.8 (a) remains true without any special set-theoretic assumptions.

4. Related questions. The Mackey problem can also be posed for $C_{c}^{*}(T)$. However the $C$ and $C^{*}$ problems are equivalent, as the following shows.

THEOREM 4.1. Let $H$ be a convex circled subset of $M_{c}(T)$. Then $H$ is $\sigma\left(M_{c}, C\right)$-compact if and only if it is $\sigma\left(M_{c}, C^{*}\right)$-compact. The same assertion is valid for countable compactness.

Proof. Assume $H$ is $\sigma\left(M_{c}, C^{*}\right)$-compact. The proof of Theorem 2.6 shows that $\operatorname{spt}(H)$ is almost $\aleph_{0}$-bounded, hence bounded in $T$. Suppose $\left(\mu_{\alpha}\right)$ is a net in $H, \mu_{0} \in H$, and $\mu_{\alpha} \rightarrow \mu_{0}$ in $\sigma\left(M_{c}, C^{*}\right)$. Let $f \in C(T)$. Then $\sup \{|f(t)|$ : $t \in \operatorname{spt}(H)\}=M<\infty$, so if $g=(-M) \vee(f \wedge M)$, then $\mu_{\alpha}(f)=\mu_{\alpha}(g) \rightarrow \mu_{0}(g)$ $=\mu_{0}(f)$. Thus the $\sigma\left(M_{c}, C^{*}\right)$ and $\sigma\left(M_{c}, C\right)$ topologies on $H$ coincide.

The proof for countable compactness is the same.

\section{REFERENCES}

1. A. G. A. Babiker, On almost discrete spaces, Mathematika 18 (1971), 163-167. MR 45 \# 5299.

2. P. Bacon, The compactness of countably compact spaces, Pacific J. Math. 32 (1970), 587-592. MR 41 \# 2624.

3. H. Buchwalter, Fonctions continues et mesures sur un espace complètement régulier, Lecture Notes in Math., vol. 331, Springer-Verlag, Berlin and New York, 1973, pp. 183-202.

4. R. C. Buck, Bounded continuous functions on a locally compact space, Michigan Math. J. 5 (1958), 95-104. MR 21 \# 4350.

5. J. B. Conway, The strict topology and compactness in the space of measures. II, Trans. Amer. Math. Soc. 126 (1967), 474-486. MR 34 \# 6503.

6. M. DeWilde and J. Schmets, Caractérisation des espaces $C(X)$ ultrabornologiques, Bull. Soc. Roy. Sci. Liège 40 (1971), 119-121. MR 45 \# 872.

7. J. Dugundji, Topology, Allyn and Bacon, Boston, Mass., 1966. MR 33 \# 1824.

8. D. H. Fremlin, D. J. H. Garling and R. Haydon, Bounded measures on topological spaces, Proc. London Math. Soc. (3) 25 (1972), 115-136. MR 49 \# 9144.

9. L. Gillman and M. Jerison, Rings of continuous functions, Univ. Ser. in Higher Math., Van Nostrand, Princeton, N. J., 1960. MR 22 \# 6994.

10. S. L. Gulden, W. M. Fleischman and J. H. Weston, Linearly ordered topological spaces, Proc. Amer. Math. Soc. 24 (1970), 197-203. MR 40 \# 3511.

11. D. Gulick, Duality theory for spaces of continuous functions (to appear).

12. Duality theory for the strict topology, Studia Math. 49 (1973/74), 195 208. MR 48 \# 12013.

13. The o-compact-open topology and its relatives, Math. Scand. 30 (1972), 159-176. MR 48 \# 9366.

14. R. Haydon, On compactness in spaces of measures and measurecompact spaces, Proc. London Math. Soc. (3) 29 (1974), 1-16. 
15. R. Haydon, Sur un problème de H. Buchwalter, C. R. Acad. Sci. Paris Sér. A-B 275 (1972), A1077-A1080. MR 47 \# 3931.

16. - Trois exemples dans la théorie des espaces de fonctions continues, $\mathrm{C} . \mathrm{R}$. Acad. Sci. Paris Sér. A-B 276 (1973), A685-A687. MR 48 \# 4675.

17. T. Isawata, On closed countably-compactifications, General Topology and Appl. 4 (1974), 143-167.

18. J. L. Kelley, I. Namioka et al., Linear topological spaces, Univ. Ser. in Higher Math., Van Nostrand, Princeton, N. J., 1963. MR 29 \# 3851.

19. J. D. Knowles, On the existence of non-atomic measures, Mathematika 14 (1967), 62-67. MR 35 \# 5568.

20. P. D. Morris and D. E. Wulbert, Functional representation of topological algebras, Pacific J. Math. 22 (1967), 323-337. MR 35 \# 4730.

21. S. E. Mosiman and R. F. Wheeler, The strict topology in a completely regular setting: relations to topological measure theory, Canad. J. Math. 24 (1972), 873-890. MR 48 \# 6909.

22. L. Nachbin, Topological vector spaces of continuous functions, Proc. Nat. Acad. Sci. U. S. A. 40 (1954), 471-474. MR 16, 156.

23. M. Rajagopalan and R. F. Wheeler, Sequential compactness of $X$ implies a completeness property for $C(X)$, Canad. J. Math. 28 (1976), 207-210.

24. M. E. Rudin, Lectures on set theoretic topology, C. B. M. S. Regional Conference Series, no. 23, Amer. Math. Soc., Providence, R. I. , 1975.

25. W. Rudin, Continuous functions on compact spaces without perfect subsets, Proc. Amer. Math. Soc. 8 (1957), 39-42. MR 19, 46.

26. H. Schaefer, Topological vector spaces, Macmillan, New York, 1966. MR 33 \# 1689.

27. F. D. Sentilles, Bounded continuous functions on a completely regular space, Trans. Amer. Math. Soc. 168 (1972), 311-336. MR 45 \# 4133.

28. T. Shirota, On locally convex vector spaces of continuous functions, Proc. Japan Acad. 30 (1954), 294-298. MR 16, 275.

29. F. D. Tall, An alternative to the continuum hypothesis and its uses in general topology (to appear).

30. V. S. Varadarajan, Measures on topological spaces, Mat. Sb. 55 (97) (1961), 35 100; English transl., Amer. Math. Soc. Transl. (2) 48 (1965), 161-228. MR 26 \# 6342.

31. S. Warner, The topology of compact convergence on continuous function spaces, Duke Math. J. 25 (1958), 265-282. MR 21 \# 1521.

32. W. A. R. Weiss, Countably compact, perfectly normal spaces may or may not be compact, Notices Amer. Math. Soc. 22 (1975), A-334. Abstract \# 75 T-G32.

33. R. F. Wheeler, Well-behaved and totally bounded approximate identities for $C_{0}(X)$, Pacific J. Math. (to appear).

DEPARTMENT OF MATHEMATICAL SCIENCES, NORTHERN ILLINOIS UNIVERSITY, DEKALB, ILLINOIS 60115 